Acknowledgement: Cooperation on data collection: All Showa University in Rheumatoid Arthritis (ASHURA) group

Disclosure of Interests: YUSUKE MIWA Grant/research support from: Mitsubishi Tanabe Pharma Corporation and AbbVie CK. Astellas Pharma Inc., Mitsubishi Tanabe Pharma Corporation, AbbVie CK, Pfizer Japan Inc., Chugai Pharmaceutical Co., Ltd., Eizai Co., Ltd, Asahi Kasei Pharm Co., Ltd, YL Biologics Ltd., Japan Blood Products Organization, Ono Pharmaceutical Co., Ltd., Nippon Kayaku Co., Ltd., and Teijin Pharma Ltd.

This work was supported by JPJS KAKENHI Grant Number JP17K09324., Kenji Sanada: None declared, Yuko Mitamura: None declared

DOI: 10.1136/annrheumdis-2019-eular.2135

\section{AB0341 REASONS FOR DROP-OUT IN RHEUMATOLOGY SPECIALTY CARE OF ELDERLY RHEUMATOID ARTHRITIS PATIENTS}

Shinichi Mizuki. Matsuyama Red Cross Hospital, The Center for Rheumatic Diseases, Matsuyama, Japan

Background: The difference between total and healthy life expectancies were 12.3 and 8.8 years for women and men respectively in 2016, in Japan. Rheumatoid arthritis (RA) not only reduces daily living activities due to joint symptoms but also deteriorates the life prognosis due to systemic inflammation. That is, patients with RA are short for both total and healthy life expectancy. In recent years, the need for rheumatologist to provide specialty medical care to elderly patients with RA has been expanding. However, some elderly patients drop out of specialty treatment and care. To date, there are little information concerning background of patients who drop out

Objectives: To investigate reasons and characteristics of elderly RA patients who drop out from rheumatology specialty medical care.

Methods: Of RA patients who had been visited our rheumatology specialty facility in 2016, we defined as drop-out when the patients did not return to the hospital in 2017 . We surveyed age, gender, disease activity, and reason for drop-out retrospectively from medical records and questionnaires.

Results: Of 2,092 patients with RA who visited to our department, 156 patients $(7.5 \%$, 95\% confidential interval: 6.4 - 8.6\%) dropped out. Among drop-out patients, 101 patients were older than 65. 37 patients (37\%) dropped out due to comorbidities including death (group C), 32 $(32 \%)$ patients were introduced certified rheumatologists near the patients' residences (group R). Twenty-two patients were due to unknown reasons, nine were due to remission, and one patient moved out to other area. Average age of both group $\mathrm{C}$ and $\mathrm{R}$ were eighty years old. Glucocorticoid user rate (C: 89\%, R: 71\%) and dose (C: $5.6 \mathrm{mg}, \mathrm{R}: 5.7 \mathrm{mg}$ ) were similar in the both two groups. Patients in group $\mathrm{C}$ showed less use of methotrexate (C: $19 \%$ vs R: $58 \%, P<0.01)$ compared with group $\mathrm{R}$ patients. Simple disease activity index was similar, however, higher modified health assessment questionnaire was observed in group C patients (C: 1.13 vs $R: 0.25, p=0.01$ ).

Conclusion: Some elderly RA patients, especially may drop out from rheumatology specialty care due to comorbidities. Regional co-management should be constructed so that elderly patients could continue receiving RA specialized care.

Disclosure of Interests: None declared

DOI: 10.1136/annrheumdis-2019-eular.1544

\section{AB0342 DEPRESSION ON PATIENTS WITH RHEUMATOID ARTHRITIS}

Simeon Monov ${ }^{1}$, Russka Shumnalieva ${ }^{2}$, Elena Miloshova ${ }^{3}$, Daniela Monova ${ }^{4}$. ${ }^{1}$ Medical University - Sofia, Department of Rheumatology, Sofia, Bulgaria; ${ }^{1}$ Medical University - Sofia, Department of Rheumatology, Sofia, Bulgaria; ${ }^{3}$ Medical Institute, Department of Internal Diseases, Sofia, Bulgaria; ${ }^{4}$ Medical Institute, Medical University - Sofia, Department of Internal Diseases, Sofia, Bulgaria

Background: Rheumatoid arthritis (RA) is a chronic representative inflammatory autoimmune disease. The association of disease activity and proinflammatory cytokines with depression has not been sufficiently investigated.

Objectives: The aim of this study is to analyze the association between disease activity and depression using Patient Help Questionnaire (PHQ-9) in patients with rheumatoid arthritis (RA). We also examined the outcome of intervention on depression score and determined the prevalence of depression and risk factors for depression and deterioration of depressive symptoms in RA patients.

Methods: 146 RA patients with a mean age of $51,3 \pm 11,2$ years were included in the study. Demographic and laboratory data were examined. Disease activity score 28-joint count C-reactive protein (DAS 28-CRP) was performed to assess disease activity of RA. PHQ-9 scores were collected at each clinic visit. Physicians assessed corresponding disease activity using Clinical Disease Activity Index (CDAI). Patients with at least moderate depression (PHQ-9 _10) were offered depression intervention, counseling or medications. $\mathrm{PHQ}-9$ was re-administered after intervention.

Results: 119 of RA patients were females, the average disease duration was $6,8 \pm 5,9$ years. Depression was diagnosed in 38 of RA patients: 18 - mild, 13 - moderate and 7 - moderately severe. Severity of depres sion positively correlated with disease activity in RA patients $(p<0.05)$. RA patients with moderate/high CDAI had significantly higher PHQ-9 than those with low CDAI $(p<0.001)$. Of 7 patients who met criteria for depression intervention, 6 were treated and 1 - declined. With treatment 5 patients had improved $\mathrm{PHQ}-9$ scores, 1 patient worsened, and 1 patient had no change in score. The risk of developing a depressive disorder is highest between 5 and 10 years of onset of the disease and depression is a better predictor of work disability than disease activity and response to treatment. Depression is associated with more pain, fatigue and impaired quality of life. Therefore, the risk to develop a depression is increased with impaired function as measured by the health assessment questionnaire (HAQ). Increased disease activity increases the risk for depression in RA. The severity of disease activity of RA, DAS 28-CRP [OR 1.75, 95\% Cl 1.08-2.64]) and severity of fatigue (OR 1.32 $95 \% \mathrm{Cl} 1.12-1.27)$ were associated with depression and deterioration of depressive symptoms in the multivariate analysis. Among the components of DAS 28-CRP, patient assessment for global health and abilities fo daily performance were more related to depression. Depression unfavorably influences the response to therapy, the rate of remission is lower and the mortality is increased in RA patients. Taken together, this indicates that it is necessary to detect a depression in patients with RA as early as possible in order to initiate appropriate treatment of depression in such cases.

Conclusion: Our study shows depression in $19,18 \%$ of patients. Correlation between disease activity and depression score is found in RA patients. Depression intervention resulted in PHQ-9 improvement in some patients, supporting the benefit of depression screening and treatment in rheumatology practice. Depression was related with the level of fatigue and high RA disease activity, which was associated with impaired ability to perform activities of daily life. Strict control of fatigue and disease activity to improve one's capacity to perform daily life activities would be important to regulate depression. Depression is common and associated with worse outcomes among patients with RA

Disclosure of Interests: None declared

DOI: 10.1136/annrheumdis-2019-eular.920

\section{AB0343 ANTIRHEUMATIC THERAPY IS NOT ASSOCIATED WITH CHANGES IN CIRCULATING N-TERMINAL PRO-BRAIN NATRIURETIC PEPTIDE (NT-PROBNP) LEVELS IN PATIENTS WITH RHEUMATOID ARTHRITIS}

Thao Nguyen ${ }^{1}$, Gia Deyab ${ }^{2}$, Morten Fagerland ${ }^{3}$, Stefan Agewall ${ }^{4}$, Gro Eilertsen ${ }^{5}$, Mark Feinberg ${ }^{6,7}$, Knut Mikkelsen $^{1}$, Øystein Førre ${ }^{8}$, Ivana Hollan ${ }^{1,7}$. ' ${ }^{1}$ Lillehammer Hospital for Rheumatic Disease, Lillehammer, Norway; ${ }^{2}$ Innlandet Hospital Trust, Department of Medical Biochemistry, Lillehammer, Norway; ${ }^{3}$ Oslo University Hospital, Oslo Centre for Biostatistics and Epidemiology, Oslo, Norway; ${ }^{4}$ Oslo University Hospital, Ulleval, Department of Cardiology, Oslo, Norway; ${ }^{5}$ The Artic University of Norway, Department of Rheumatology, Tromsø, Norway; ${ }^{6}$ Harvard Medical School, Boston, United States of America; ${ }^{7}$ Brigham and Women's Hospital, Department of Cardiology, Boston, United States of America; ${ }^{8}$ University of Oslo, Oslo, Norway

Background: Patients with rheumatoid arthritis (RA) are predisposed to impaired cardiac function and heart failure (HF). While the pathophysiology has not been fully elucidated yet, inflammation is suspected to play an important role. However, the impact of disease-modifying antirheumatic drugs on cardiac dysfunction in RA remains controversial. Although anti-inflammatory drugs might have protective effects, some of them, i.e. tumour necrosis factor inhibitors (anti-TNF), might also negatively influence cardiac function. Serum NT-proBNP (s-NT-proBNP) is used as a biomarker of cardiac function, and levels $\leq 125 \mathrm{ng} / \mathrm{L}$ with high probability exclude $\mathrm{HF}^{1}$.

Objectives: To examine effects of methotrexate (MTX) and anti-TNF regimens on s-NT-proBNP in patients with active RA, and to assess associations between s-NT-proBNP and endothelial function (EndoF). 
Methods: From the observational PSARA study, we examined 64 RA patients starting with MTX monotherapy $(n=34)$ or anti-TNF with MTX comedication $(n=30)$ due to active disease. All patients starting with antiTNF regimens had been previously unsuccessfully treated with MTX. sNT-proBNP (ELISA), EndoF (measured by finger plethysmography), and other laboratory and clinical parameters were evaluated at baseline and after 6 weeks and 6 months of treatment.

Results: Median age was 57 years (range 28-79), and 73\% were women. $17(27 \%)$ patients had CVD (history of angina, MI, heart surgery, PTA, cerebrovascular disease, thromboembolism, aortic aneurysm, peripheral artery disease). None of the patients had known/symptomatic HF. There were no statistically significant differences between s-NT-proBNP levels at baseline (median $2241 \mathrm{ng} / \mathrm{L}$ [IQR 9002]) and after 6 weeks (median $2300 \mathrm{ng} / \mathrm{L}$, [IQR 8960]) and 6 months (median $2358 \mathrm{ng} / \mathrm{L}$ [IQR 7772]) of antirheumatic therapy $(\mathrm{p}=0.992$ and $\mathrm{p}=0.528$, respectively). There were no significant differences in the effects of MTX monotherapy and anti-TNF regimens on sNT-proBNP levels ( $p_{\text {baseline-6weeks }}=0.779$; $p_{\text {baseline- } 6 \text { months }}=0.421$ ).

At baseline, 57 (89\%) patients had s-NT-proBNP>125 ng/L, and 44 $(69 \%)$ had high s-NT-proBNP levels (s-NT-proBNP $>450 \mathrm{ng} / \mathrm{L}$ in patients $<50$ years old and $>900 \mathrm{ng} / \mathrm{L}$ in patients $>50$ years old), and these frequencies did not significantly change with antirheumatic treatment. s-NTproBNP was not related to EndoF.

Conclusion: A large proportion of RA patients without known HF had elevated s-NT-proBNP levels, which might indicate subclinical impairment of cardiac function. s-NT-proBNP levels were not influenced by six-month MTX and/or anti-TNF treatment. Thus, in contrast to some previous studies, our data does not support the notion that anti-inflammatory treatment protects against HF, and that anti-TNF treatment has negative effect on cardiac function in RA. Nevertheless, definitive conclusions cannot be drawn by our study, e.g. due to limitations of s-NT-proBNP as surrogate marker of HF. Cardiac function in terms of s-NT-proBNP levels was not related to EndoF

\section{REFERENCES}

[1] Ponikowski P, et al. European journal of heart failure. 2016;18:891-975.

Disclosure of Interests: None declared

DOI: 10.1136/annrheumdis-2019-eular.3533

\section{AB0344 COMPARATIVE ANALYSIS OF SIDE EFFECTS BETWEEN PATIENTS TREATED WITH AND WITHOUT CORTICOSTEROID AT DMARDS-STARTING POINT SINGLE CENTER RETROSPECTIVE REAL WORLD DATA ANALYSIS}

Michihiro Ogasawara ${ }^{1}$, Yosuke Shimada ${ }^{2}$, Yoshiyuki Abe ${ }^{1}$, Ken Yamaji ${ }^{1}$ Satoshi Hori ${ }^{3}$, Naoto Tamura'. ' 'Juntendo University Faculty of Medicine, Department of Internal Medicine and Rheumatology, Tokyo, Japan; ${ }^{2}$ SECOM Co., Ltd., Intelligent Systems Laboratory, Tokyo, Japan; ${ }^{3}$ Juntendo University Graduate School, Medical Informatics Department, Tokyo, Japan

Background: In the EULAR recommendation on rheumatoid arthritis (RA) treatment, short-term steroid combination should be considered at the start of DMARDs treatment. In this study, we analyzed the present condition of steroid combined use in Japan and the actual situation of side effects in recent years.

Objectives: RA patients who started treatment with DMARDs at our hospital during July 2008 to April 2018,

Methods: A new incidence of hypertension (HTN), diabetes (DIB), dyslipidemia (HLP), infection (INF) were compared between the two groups of steroid combined use group (D_PSL) and noncombined group (D_nonPSL) and analyzed by Cox regression analysis. The cases had there complications before the start of RA treatment were excluded from the analysis.

Results: The number of cases of analysis was 573 (D PSL $=216$, D_nonPSL $=357$, average observation period: $5.22 \pm 2.70$ year), and the new incidence of each complication was INF 50/561 (D PSL $16.2 \%$ vs. D_nonPSL 4.56\%[16/351], $p=0.000094)$, HTN 48/527 cases $(13.3 \%[25 / 188]$ vs.6.78\%[23/339], $P=0.0148)$, DIB $24 / 558$ (6.16\%[13/211] vs. $3.17 \%[11 /$ 347], $\mathrm{p}=0.136), \mathrm{HLP} 72 / 542(15.8 \%[32 / 202]$ vs. $11.8 \%[40 / 340], \mathrm{p}=$ 0.198). The incidence of INF was high in D_PSL group in both groups over 65 and under 65 years old $(p=0.00658, p=0.00267)$, HTN was high in D_PSL group only in group over 65 years old $(p=0.0276$, $p=0.0554$ ). Average starting amount of steroids was $6.07 \pm 10.2 \mathrm{mg}$, cases of non-withdrawal of steroid at $1,2,3$ years later is $79.7 \%, 72.2 \%$ and $66.3 \%$ respectively.

Conclusion: Combined use of steroid at the start of DMARDs increased the rate of mortality of infection requiring hospitalization and hypertension.
More than half of them were difficult cases of withdrawal of steroids. From the viewpoint of side effect, it is necessary to study the optimization of steroid therapy in the future.

Disclosure of Interests: Michihiro Ogasawara: None declared, Yosuke Shimada: None declared, Yoshiyuki Abe: None declared, Ken Yamaji: None declared, Satoshi Hori: None declared, Naoto Tamura Grant/research sup port from: Astellas Pharma Inc., Asahi Kasei Pharma, AYUMI Pharmaceutical Co., Chugai Pharmaceutical Co. LTD, Eisai Inc., :Takeda Pharmaceutical Company Ltd., Speakers bureau: Janssen Pharmaceutical K.K., Bristol-Myers Squibb K.K., :Mitsubishi Tanabe Pharma Co.

DOI: 10.1136/annrheumdis-2019-eular.1588

\section{AB0345 1 A PILOT ATTEMPT TO USE THE CONSTANT GENETIC MARKERS IN CARDIOVASCULAR RISK STRATIFICATION IN RHEUMATOID ARTHRITIS}

Vitaly Omelchenko ${ }^{1}$, Elena Letyagina ${ }^{1}$, Tatyana Pospelova ${ }^{2}$, Maxim Korolev ${ }^{1}$ ${ }^{1}$ RICEL - Branch of ICandG SB RAS, Novosibirsk, Russian Federation; ${ }^{2} N S M U$, Department of Therapy, Hematology and Transfusiology, Novosibirsk, Russian Federation

Background: Rheumatoid arthritis (RA) is associated with early atherosclerosis and high mortality. Routine riskometers developed for a common population usually underestimate a true cardiovascular (CV) risk in RApatients. For early prevention additional criteria is needed to evaluate $\mathrm{CV}$ risk in RA-patients. It's known, that early CV accidents in relatives relate to elevated risk. Nevertheless, specific constant genetic markers don't use in risk stratification. Single nucleotide polymorphisms in regulatory regions of genes, participating in immune interaction, can potentially play a role in progression atherosclerosis and used in CV-prevention algorithms.

Objectives: to develop the model for cardiovascular risk stratification in RA-patients including constant genetic markers.

Methods: Two hundred and twelve Caucasian patients with RA (age 58.0 yrs [48.3; 65.0]; DAS28 $4.96[3.86 ; 5.85])$ were included in our study. Patients had American College of Rheumatology (ACR)-defined RA (1987 classification criteria). All patients gave written informed consent before enrollment. Traditional and "non-traditional" (e.g. RA-associated factors) were analyzed [1]. Carotid atherosclerotic plaques had been found by ultrasonography. Single nucleotide polymorphisms were determined by restriction fragment length polymorphism (TNFA G-308A (rs1800629), TNFA C-863A (rs1800630), TNFA G-238A (rs361525), IL6 G-174C (rs1800795), VEGFA C-2578A (rs699947), MMP3 5-1171 6A (rs 3025058), MMP9 -1562T (rs3918242)) and polymerase chain reaction (IL1B $\quad$ T-31C (rs1143627), IL4 C-590T (rs2243250), IL10 C-592A (rs1800872), IL10 A-1082G (rs1800896), VEGFA C+936T (rs3025039), MMP2 C-1306T (rs2438650)). Descriptive statistics, Chi-squared test, logistic regression with Wald statistics were used for data analysis. Results are presented as median and 25th/75th percentiles (Me [25th percentile; 75th percentile]).

Results: Atherosclerotic plaques (API) were found in 59 (27.8\%) patients API revealed were strongly associated with age $(66.0$ yrs $[59.0 ; 73.0]$ with $\mathrm{API}$ vs 55.0 yrs $[42.0 ; 61.0]$ without $\mathrm{API}, \mathrm{p}<0.001)$ and $\operatorname{sex}(51.6 \%$ for man vs $23.8 \%$ for woman, $\mathrm{p}<0.001)$. Then, a logistic regression was performed to determine which variables analyzed are predictors of a carotid atherosclerotic lesion. Regression results had demonstrated that the model was statistically reliable in distinguishing between patients with AP and without $[-2$ Log Likelihood $=146.53, p<001]$. The model correctly classified $85.3 \%$ of cases. The Wald statistics showed that at least 6 parameters were principal - age $(B=0.123, \quad p<0.001)$, hypertension $(B=3.114, p<0.001)$, smoke $(B=2.167, p<0.001)$, positive rheumatoid factor $(\mathrm{B}=1.674, \mathrm{p}=0.038)$, body mass index $(\mathrm{B}=-0.098,0.019)$ and recessive allele in TNFA $C$-863A $(B=1.338, p=0.005)$. Using parameters obtained the ROC-curve was constructed. Area under ROC curve were 0.900 (SE $0.022 ; 95 \% \mathrm{Cl} 0.857-0.942, \mathrm{P}<0.001)$. Sensitivity and specificity calculated by Youden's Index were $79.7 \%$ and $87.5 \%$, respectively.

Conclusion: Age, hypertension, smoke, positive rheumatoid factor, low body mass index and recessive allele in TNFA C-863A were associated with carotid atherosclerosis.

\section{REFERENCES}

[1] Amaya-Amaya J. et al. Novel risk factors for cardiovascular disease in rheumatoid arthritis//Immunol. Res. 2013. Vol. 56, No 2-3. P. 267-286

Disclosure of Interests: None declared

DOI: 10.1136/annrheumdis-2019-eular.3544 